\title{
Effects of heavy Majorana neutrinos on lepton flavor violating processes
}

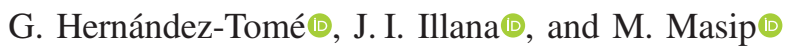 \\ CAFPE and Departamento de Física Teórica y del Cosmos, Universidad de Granada, \\ E18071 Granada, Spain \\ G. López Castro® and P. Roig \\ Departamento de Física, Centro de Investigación y de Estudios Avanzados del Instituto Politécnico \\ Nacional, Apartado Postal 14-740, 07000 México D.F., México
}

(Received 15 January 2020; accepted 17 March 2020; published 10 April 2020)

\begin{abstract}
The observation of lepton flavor violating processes at colliders could be a clear signal of a nonminimal neutrino sector. We define a 5-parameter model with a pair of $\mathrm{TeV}$ fermion singlets and arbitrary mixings with the three active neutrino flavors. Then we analyze several flavor violating transitions $\left(\ell \rightarrow \ell^{\prime} \gamma\right.$, $\ell^{\prime} \ell^{\prime \prime} \bar{\ell}^{\prime \prime \prime}$, or $\mu-e$ conversions in nuclei) and $Z \rightarrow \bar{\ell} \ell^{\prime}$ decays induced by the presence of heavy neutrinos. In particular, we calculate all the one-loop contributions to these processes and present their analytic expressions. We focus on the genuine effects of the heavy Majorana masses, comparing the results in that case with the ones obtained when the two heavy neutrinos define a Dirac field. Finally, we use our results to update the bounds on the heavy-light mixings in the neutrino sector.
\end{abstract}

DOI: $10.1103 /$ PhysRevD.101.075020

\section{INTRODUCTION}

In the original formulation of the standard model (SM) [1-3], the lepton flavor and the lepton number are accidentally conserved quantities due to the assumption of massless neutrinos. However, this framework must be extended to account for the well-established evidence of neutrino oscillations [4-6], which implies nonzero masses and mixings for the active neutrinos. A possible minimal extension is the so-called $\nu \mathrm{SM}$ [7], which adds righthanded components (gauge singlets) for the three neutrino families and generates Dirac masses via Yukawa couplings with the Higgs doublet, just like for all the other fermions. In the $\nu \mathrm{SM}$, the mixing in the leptonic sector is described by a $3 \times 3$ unitary matrix called the Pontecorvo-MakiNakagawa-Sakata (PMNS) matrix [8,9], analogous to the Cabibbo-Kobayashi-Maskawa (CKM) matrix of the quark sector $[10,11]$. Nevertheless, the $\nu \mathrm{SM}$ requires extremely tiny Yukawa couplings to explain the observed masses, which suggests that other mechanism may be at work. If, in addition to the Dirac mass terms $\left(m_{D}\right)$ that combine them with the active neutrinos, the singlets have Majorana masses $\left(m_{M}\right)$ that define a new scale, then the tiny neutrino masses appear naturally for a very large value of $m_{M}$ (i.e.,

Published by the American Physical Society under the terms of the Creative Commons Attribution 4.0 International license. Further distribution of this work must maintain attribution to the author(s) and the published article's title, journal citation, and DOI. Funded by SCOAP ${ }^{3}$.
$\left.m_{M} \gg m_{D}\right)$. In this seesaw mechanism [12-14] the new mass terms break lepton number. The physical states after diagonalization of the mass matrix include light $(\nu)$ and heavy $(N)$ sectors of Majorana neutrinos with masses

$$
m_{\nu} \approx m_{D}^{2} / m_{M}, \quad m_{N} \approx m_{M} \gg m_{\nu} .
$$

Like in the $\nu \mathrm{SM}$, in this model the mixings among the three active families may be large, as required from oscillation experiments, but the mixing with the heavy fields is of order

$$
s_{\nu} \approx m_{D} / m_{M} \approx \sqrt{m_{\nu} / m_{N}} .
$$

Both in the $\nu \mathrm{SM}$ and this high scale (type I) seesaw model, the rate of lepton flavor violating (LFV) processes at colliders is suppressed by a factor of $\left(m_{\nu} / E\right)^{2}$, being $E$ the scale of the process. In the second scenario LFV can also be mediated by the neutrinos in the heavy sector, but the heavy-light mixing implies then a suppression of order of $\left(E / m_{N}\right)^{2}$, equally small. In particular, the LFV decays $\ell \rightarrow \ell^{\prime} \gamma, \ell \rightarrow \ell^{\prime} \ell^{\prime \prime} \bar{\ell}^{\prime \prime \prime}$, and $Z \rightarrow \bar{\ell} \ell^{\prime}$, where $\ell, \ell^{\prime}, \ldots$ denote the usual charged leptons $(\tau, \mu, e)$ will have a branching ratio below $10^{-50}$ [15-21]. It is then apparent that any experimental observation of flavor violation involving charged leptons (cLFV) would unambiguously imply the existence of new physics at the $\mathrm{TeV}$ scale in an extended neutrino sector [22-28].

Well-motivated variants of the two minimal models described above include the inverse seesaw $[29,30]$ or 
the linear seesaw [31]. These scenarios allow for arbitrary masses in the heavy neutrino sector and then unsuppressed heavy-light mixings, constrained only by the experimental limits. They are usually known as low-scale seesaw models, although the masses in both sectors are not necessarily correlated. They are justified by approximate symmetries or some ansatz on the neutrino mass matrix that relaxes the restriction in Eq. (2). This type of models may be adequate in scenarios like little Higgs (the heavy Majorana in seesaw models would introduce quadratic corrections to the Higgs mass [32-34]), supersymmetry [35-37], TeV gravity models (with the cutoff right above that scale) [38,39], or composite Higgs models [40]. In the next section we present a simple model that captures all the relevant effects that may appear in cLFV processes induced by the presence of heavy neutrinos.

Another possibility in these scenarios that is interesting from the phenomenological point of view is to test the Dirac or Majorana nature of the neutrinos in the heavy sector through lepton number $(L)$ violating processes with $\Delta L=2$. Apart from the long-explored neutrinoless double-beta decay [41,42], this has been undertaken in tau decays $\tau^{-} \rightarrow \ell^{+} M_{1}^{-} M_{2}^{-}$( $M_{1}, M_{2}=\pi, K$ mesons) [43-45], meson decays $M_{1}^{+} \rightarrow \ell_{1}^{+} \ell_{2}^{+} M_{2}^{-}$and hyperon decays, like $\Sigma^{-} \rightarrow \Sigma^{+} e^{-} e^{-}, \Sigma^{-} \rightarrow p \mu^{-} \mu^{-}$[46,47], etc. All these studies are based on scenarios where the new sterile Majorana neutrinos have non-negligible mixings and some of them require masses low enough to be produced on-shell (resonant-enhancement).

Currently there is no evidence for cLFV, but intense experimental efforts have provided strong limits on an extensive list of processes; some of them are reported in Table I. The sensitivity to these transitions will be considerably improved in near-future experiments. The MEG-II and Mu3e experiments will reach branching ratios of order $6 \times 10^{-14}$ [48] and $10^{-16}$ [49] for $\mu \rightarrow e \gamma$ and $\mu \rightarrow e e \bar{e}$, respectively, whereas the expected bounds from PRISM and COMET will be near $10^{-18}$ [50] for $\mu-e$ (Ti) conversion and $10^{-17}$ [51] for $\mu-e$ (Al). For the third family, the bounds on the $\tau \rightarrow \ell^{\prime} \gamma$ and $\tau \rightarrow \ell^{\prime} \ell^{\prime \prime} \bar{\ell}^{\prime \prime \prime}$ branching ratios could be improved by two orders of magnitude at Belle-II when the experiment achieves its maximum luminosity [52,53]. LHCb has already set a stringent limit (competitive with the present ones at Belle) of $4.6 \times 10^{-8}$ [54] on the $\tau \rightarrow \mu \mu \bar{\mu}$ process. In its highluminosity phase the LHC is expected to improve this bound by one order of magnitude. Additionally, the possibility of running at the $Z$ pole in the electron-positron version of a Future Circular Collider (FCC-ee) $[55,56]$ or in the Circular Electron Positron Collider (CEPC) [57] would improve the current limits on $Z \rightarrow \bar{\ell} \ell^{\prime}$ by about four orders of magnitude. Finally, the expected sensitivity of the HL-LHC $\left(3000 \mathrm{fb}^{-1}\right)$ will be around $5 \times 10^{-4}$ for both the $h \rightarrow e \tau$ and $h \rightarrow \mu \tau$ branching fractions [58].
TABLE I. Present limits and future sensitivities for the branching ratios or capture rates of several LFV processes. We denote $Z \rightarrow \ell_{1} \ell_{2} \equiv Z \rightarrow \bar{\ell}_{1} \ell_{2}+\ell_{1} \bar{\ell}_{2}$, and similarly for $h$ decays. For a more extensive list including hadronic modes see $[59,60]$.

\begin{tabular}{lcc}
\hline \hline Reaction & Present limit $90 \%$ C.L. & Future sensitivity \\
\hline$\mu \rightarrow e \gamma$ & $4.2 \times 10^{-13}[61]$ & $6 \times 10^{-14}[48]$ \\
$\mu-e(\mathrm{Au})$ & $7.0 \times 10^{-13}[62]$ & $\ldots$ \\
$\tau \rightarrow e \gamma$ & $3.3 \times 10^{-8}[63]$ & $3 \times 10^{-9}[52]$ \\
$\tau \rightarrow e e \bar{e}$ & $2.7 \times 10^{-8}[64]$ & $(2-5) \times 10^{-10}[52]$ \\
$\tau \rightarrow e \mu \bar{\mu}$ & $2.7 \times 10^{-8}[64]$ & \\
$\tau \rightarrow e e \bar{\mu}$ & $1.5 \times 10^{-8}[64]$ & $10^{-16}[49]$ \\
$\mu \rightarrow e e \bar{e}$ & $1.0 \times 10^{-12}[65]$ & $10^{-18}[50]$ \\
$\mu-e(\mathrm{Ti})$ & $4.3 \times 10^{-12}[62]$ & $10^{-9}[52]$ \\
$\tau \rightarrow \mu \gamma$ & $4.4 \times 10^{-8}[63]$ & \\
$\tau \rightarrow \mu \mu \bar{\mu}$ & $2.1 \times 10^{-8}[64]$ & \\
$\tau \rightarrow \mu e \bar{e}$ & $1.8 \times 10^{-8}[64]$ & \\
$\tau \rightarrow \mu \mu \bar{e}$ & $1.7 \times 10^{-8}[64]$ & \\
\hline
\end{tabular}

\begin{tabular}{lcc}
\hline \hline Reaction & Present limit 95\% C.L. & Future sensitivity \\
\hline$Z \rightarrow \mu e$ & $7.3 \times 10^{-7}[66]$ & $10^{-10}[55]$ \\
$Z \rightarrow \tau e$ & $9.8 \times 10^{-6}[67]$ & $10^{-9}[55]$ \\
$Z \rightarrow \tau \mu$ & $1.2 \times 10^{-5}[68]$ & $10^{-9}[55]$ \\
$h \rightarrow \mu e$ & $3.4 \times 10^{-4}[69]$ & $\ldots$ \\
$h \rightarrow \tau e$ & $6.2 \times 10^{-3}[70]$ & $5 \times 10^{-4}[58]$ \\
$h \rightarrow \tau \mu$ & $2.5 \times 10^{-3}[70]$ & \\
\hline \hline
\end{tabular}

In this work we focus on the most phenomenologically relevant cLFV observables in the framework of low-scale seesaw scenarios. In Sec. II we introduce a model for the mixings of the active neutrinos with two singlet fermions defining Majorana fields. The mass splitting between these heavy fields parametrizes the breaking of lepton number; when the splitting vanishes the heavy sector reduces to a single Dirac neutrino. In Sec. III we provide detailed expressions for the amplitudes and decay rates of the processes under consideration. In Sec. IV we use these observables to derive constraints on the heavy-light mixing angles as a function of the masses of the two heavy states. Our conclusions are given in Sec. V.

\section{A MODEL FOR THE HEAVY-LIGHT NEUTRINO MIXING}

As mentioned before, in the usual type-I seesaw model with one Majorana singlet per family the heavy-light mixings are correlated with the neutrino masses: to obtain $m_{\nu}<1 \mathrm{eV}$ with $m_{D} \approx 1 \mathrm{GeV}$ we need $m_{M}>10^{9} \mathrm{GeV}$, and this implies negligible heavy-light mixings, $s_{\nu}<10^{-9}$. As it is well known by the practitioners, however, this is no longer the case when the singlet fermions are introduced in pairs (see [71] for a recent review). In particular, all the heavy-light mixing effects can be captured by considering a model with just one extra pair. Let us see how this works. 
We take two bispinors $N$ and $N^{c}$ of left-handed chirality (undotted), sterile and with opposite lepton number, and define the four-spinors

$N_{L}=\left(\begin{array}{c}N \\ 0\end{array}\right) ; \quad N_{R}=\left(\begin{array}{c}0 \\ \bar{N}^{c}\end{array}\right) ; \quad \nu_{L i}=\left(\begin{array}{c}\nu_{i} \\ 0\end{array}\right)$,

where $\nu_{i=e, \mu, \tau}$ are the SM neutrinos. After the breaking of the electroweak symmetry the SM charged leptons get masses through Yukawa interactions with the Higgs field; the left-handed mass eigenstates are obtained after a unitary transformation,

$$
\ell_{L i} \rightsquigarrow \sum_{j=1}^{3} U_{i j}^{\ell} \ell_{L j},
$$

that we also perform in the space of the three active neutrinos $\nu_{L i}$. Then we assume that in this basis the 5 Majorana fields $\chi_{i}=\chi_{L i}+\chi_{L i}^{c}$ with $\chi_{L} \equiv\left(\nu_{L 1}, \nu_{L 2}, \nu_{L 3}, N_{L}, N_{R}^{c}\right)$ have mass terms

$$
\mathcal{L}_{M}=-\frac{1}{2} \overline{\chi_{L}^{c}} \mathcal{M} \chi_{L}+\text { H.c., }
$$

with

$$
\mathcal{M}=\left(\begin{array}{ccccc}
0 & 0 & 0 & 0 & m_{1} \\
0 & 0 & 0 & 0 & m_{2} \\
0 & 0 & 0 & 0 & m_{3} \\
0 & 0 & 0 & 0 & M \\
m_{1} & m_{2} & m_{3} & M & \mu
\end{array}\right)
$$

Notice that we have ordered the fields according to their lepton number (positive for the first four neutrinos), and that the Majorana mass $\mu$ corresponds to the neutrino with negative lepton number (the fifth one). The mass eigenstates are obtained diagonalizing this symmetric matrix by an orthogonal transformation and applying a field redefinition $\left(\chi_{L 4} \rightarrow i \chi_{L 4}\right)$ to guarantee real and positive mass eigenvalues. Three eigenvalues are zero $\left(m_{\chi_{1,2,3}}=0\right)$ and the other two are

$$
\begin{aligned}
& m_{\chi_{4}}=\frac{1}{2}\left(\sqrt{4\left(m_{1}^{2}+m_{2}^{2}+m_{3}^{2}+M^{2}\right)+\mu^{2}}-\mu\right), \\
& m_{\chi_{5}}=\frac{1}{2}\left(\sqrt{4\left(m_{1}^{2}+m_{2}^{2}+m_{3}^{2}+M^{2}\right)+\mu^{2}}+\mu\right) .
\end{aligned}
$$

Defining $m \equiv \sqrt{m_{1}^{2}+m_{2}^{2}+m_{3}^{2}}$ and $M^{\prime} \equiv \sqrt{m^{2}+M^{2}}$, the mass eigenstates are given by the replacement

$$
\chi_{L i} \rightsquigarrow \sum_{j=1}^{5} U_{i j}^{\nu} \chi_{L j},
$$

where the mixing matrix reads

$$
U^{\nu}=\left(\begin{array}{ccccc}
-\frac{m_{2}}{\sqrt{m_{1}^{2}+m_{2}^{2}}} & -\frac{m_{1} m_{3}}{m \sqrt{m_{1}^{2}+m_{2}^{2}}} & -\frac{m_{1} M}{m M^{\prime}} & -i \frac{m_{1} m_{\chi_{5}}}{M^{\prime} \sqrt{m_{\chi_{5}}^{2}+M^{\prime 2}}} & \frac{m_{1}}{\sqrt{m_{\chi_{5}}^{2}+M^{\prime 2}}} \\
\frac{m_{1}}{\sqrt{m_{1}^{2}+m_{2}^{2}}} & -\frac{m_{2} m_{3}}{m \sqrt{m_{1}^{2}+m_{2}^{2}}} & -\frac{m_{2} M}{m M^{\prime}} & -i \frac{m_{2} m_{\chi_{5}}}{M^{\prime} \sqrt{m_{\chi_{5}}^{2}+M^{\prime 2}}} & \frac{m_{2}}{\sqrt{m_{\chi_{5}}^{2}+M^{\prime 2}}} \\
0 & \frac{\sqrt{m_{1}^{2}+m_{2}^{2}}}{m} & -\frac{m_{3} M}{m M^{\prime}} & -i \frac{m_{3} m_{\chi_{5}}}{M^{\prime} \sqrt{m_{\chi_{5}}^{2}+M^{\prime 2}}} & \frac{m_{3}}{\sqrt{m_{\chi_{5}}^{2}+M^{\prime 2}}} \\
0 & 0 & \frac{m}{M^{\prime}} & -i \frac{M m_{\chi_{5}}}{M^{\prime} \sqrt{m_{\chi_{5}}^{2}+M^{\prime 2}}} & \frac{M^{\prime}}{\sqrt{m_{\chi_{5}}^{2}+M^{\prime 2}}} \\
0 & 0 & 0 & i \frac{M^{\prime}}{\sqrt{m_{\chi_{5}}^{2}+M^{\prime 2}}} & \frac{m_{\chi_{5}}}{\sqrt{m_{\chi_{5}}^{2}+M^{\prime 2}}}
\end{array}\right) .
$$

Several comments are in order:

(i) $\mu$ is the only mass parameter breaking lepton number. This parameter defines the mass splitting of the two heavy Majorana neutrinos: when $\mu=0$ both states form a heavy Dirac neutrino singlet of mass $M^{\prime}$.

(ii) The two heavy neutrinos $\left(N_{1,2} \equiv \chi_{4,5}\right)$ have components of order $m_{i} / M$ along the corresponding active neutrinos, where $m_{i}=Y_{\nu_{i}} v / \sqrt{2}$ are Dirac mass terms coming from Yukawa couplings with the SM Higgs doublet. If the heavy fields have $\mathrm{TeV}$ masses, the heavy-light mixings can be as large as $\sim 0.1$ for couplings of order one.

(iii) The three (mostly) active neutrinos $\left(\chi_{1,2,3}\right)$, to be identified with the light neutrinos $\left(\nu_{1,2,3}\right)$ observed so far, are exactly massless. A deformation of the pattern in Eq. (6) with a Majorana mass $\mu^{\prime}$ for $N_{L}$ (like in inverse seesaw models) would imply that one of these neutrinos gets a mass $m_{\nu} \approx \mu^{\prime}(m / M)^{2}$. Since $m_{\nu}<1 \mathrm{eV}$, however, the new term $\mu^{\prime}$ must be small and have no effect on flavor physics (it does not change the heavy-light mixings). An analogous 
argument applies to possible Dirac mass terms $m_{i}^{\prime}$ in the fourth row/column.

(iv) The pattern that we propose must be understood as approximate: these 5 entries are the dominant mass terms, and any deformation must respect that the third neutrino gets a mass below $1 \mathrm{eV}$ (i.e., it must be much smaller than these 5 terms). Notice also that no symmetry protects the entries assumed to be zero, and that loop corrections will actually introduce contributions to all of them (see [71]). The pattern must then be established at the loop level where we work, which may require the addition of tree level terms canceling radiative corrections. In particular, large values of $\mu$ would induce 1-loop values of $\mu^{\prime}$ that must be canceled to obtain the proposed pattern. This fine tuning disappears for $\mu \rightarrow 0$, when the resulting pattern is justified by the conservation of lepton number.

(v) The generation of small masses and light-light mixings for the three active neutrinos would require the addition of extra singlets. This could be accommodated with the usual mechanisms (in $\nu \mathrm{SM}$ or Type I seesaw models) or through the deformations described above (in inverse seesaw models). In any case, it will not introduce sizeable heavy-light mixings.

We will trade the five arbitrary mass parameters in Eq. (10) for the masses of the two heavy neutrinos and three heavy-light mixings,

$m_{N_{1}} \equiv m_{\chi_{4}}, \quad m_{N_{2}} \equiv m_{\chi_{5}}, \quad s_{\nu_{i}} \equiv \frac{m_{i}}{\sqrt{m_{N_{1}} m_{N_{2}}}}$.

The $5 \times 5$ matrix $U_{i j}^{\nu}$ above will introduce tree-level charged and neutral currents involving neutrinos:

$$
\begin{gathered}
\mathcal{L}_{W^{ \pm}}=-\frac{g}{\sqrt{2}} W_{\mu}^{-} \sum_{i=1}^{3} \sum_{j=1}^{5} B_{i j} \bar{e}_{i} \gamma^{\mu} P_{L} \chi_{j}+\text { H.c., } \\
\mathcal{L}_{Z}=-\frac{g}{4 c_{W}} Z_{\mu} \sum_{i, j=1}^{5} \bar{\chi}_{i} \gamma^{\mu}\left(C_{i j} P_{L}-C_{i j}^{*} P_{R}\right) \chi_{j}, \\
\mathcal{L}_{G^{ \pm}}=-\frac{g}{\sqrt{2} M_{W}} G^{-} \sum_{i=1}^{3} \sum_{j=1}^{5} B_{i j} \\
\times \bar{\ell}_{i}\left(m_{\ell_{i}} P_{L}-m_{\chi_{j}} P_{R}\right) \chi_{j}+\text { H.c. }
\end{gathered}
$$

where $G^{ \pm}$is the charged would-be-Goldstone field, $g$ is the weak coupling constant, $c_{W}=\cos \theta_{W}$ and $P_{L, R}=\frac{1}{2}\left(1 \mp \gamma_{5}\right)$ are the left and right-handed projectors, respectively. Notice that in Eq. (13), the neutral current induced by the Majorana states involves couplings of different flavors with both left and right-handed components. ${ }^{1}$ The

\footnotetext{
${ }^{1}$ For the case of heavy left-handed neutrinos being sequential Dirac (active) neutrinos, replace $B_{i j} \rightarrow U_{i j}^{\nu}, C_{i j} \rightarrow \delta_{i j}, C_{i j}^{*} \rightarrow 0$.
}

dimension of the rectangular $B$ mixing matrix is $3 \times 5$, whereas $C$ is a $5 \times 5$ matrix,

$$
B_{i j}=\sum_{k=1}^{3} \delta_{i k} U_{k j}^{\nu}, \quad C_{i j}=\sum_{k=1}^{3}\left(U_{k i}^{\nu}\right)^{*} U_{k j}^{\nu} .
$$

One can see that the elements of these matrices involving heavy neutrinos can be expressed in terms of heavy-light mixings and the squared mass ratio $r=m_{N_{2}}^{2} / m_{N_{1}}^{2}$ as

$$
\begin{gathered}
B_{k N_{1}}=-\frac{i r^{\frac{1}{4}}}{\sqrt{1+r^{\frac{1}{2}}}} s_{\nu_{k}}, \quad B_{k N_{2}}=\frac{1}{\sqrt{1+r^{\frac{1}{2}}}} s_{\nu_{k}}, \\
C_{N_{1} N_{1}}=\frac{r^{\frac{1}{2}}}{1+r^{\frac{1}{2}}} \sum_{k=1}^{3} s_{\nu_{k}}^{2}, \quad C_{N_{2} N_{2}}=\frac{1}{1+r^{\frac{1}{2}}} \sum_{k=1}^{3} s_{\nu_{k}}^{2}, \\
C_{N_{1} N_{2}}=-C_{N_{2} N_{1}}=\frac{i r^{\frac{1}{4}}}{1+r^{\frac{1}{2}}} \sum_{k=1}^{3} s_{\nu_{k}}^{2} .
\end{gathered}
$$

These are the same as in $[19,72]$ up to an irrelevant global phase for $B$. In addition, the matrices $B$ and $C$ satisfy some identities that are essential to keep the renormalizability of the model:

$$
\begin{aligned}
& \sum_{k=1}^{5} B_{i k} B_{j k}^{*}=\delta_{i j}, \quad \sum_{k=1}^{3} B_{k i}^{*} B_{k j}=\sum_{k=1}^{5} C_{i k} C_{j k}^{*}=C_{i j}, \\
& \sum_{k=1}^{5} B_{i k} C_{k j}=B_{i j},
\end{aligned}
$$

$$
\sum_{k=1}^{5} m_{\chi_{k}} C_{i k} C_{j k}=\sum_{k=1}^{5} m_{\chi_{k}} B_{i k} C_{k j}^{*}=\sum_{k=1}^{5} m_{\chi_{k}} B_{i k} B_{j k}=0 .
$$

\section{LFV PROCESSES}

We now present the amplitudes and decays widths or transition rates for the LFV processes $\ell \rightarrow \ell^{\prime} \gamma, Z \rightarrow \bar{\ell} \ell^{\prime}$, $\ell \rightarrow \ell^{\prime} \ell^{\prime \prime} \bar{\ell}^{\prime \prime \prime}$, and $\mu-e$ conversion in nuclei. All of them involve the effective interaction of a neutral vector boson with a pair of on-shell fermions, $V \ell \ell^{\prime}(V=\gamma, Z)$, through a loop with Majorana neutrinos. Since the $W$ couples only to left-handed fields, the effective $V \ell \ell^{\prime}$ vertices $\left(\ell \neq \ell^{\prime}\right)$ can be written in terms of the following form factors:

$$
\begin{gathered}
i \Gamma_{\mu}^{\gamma}\left(q^{2}\right)=i e\left[F_{L}^{\gamma}\left(q^{2}\right) \gamma_{\mu} P_{L}+i 2 F_{M}^{\gamma}\left(q^{2}\right) P_{R} \sigma_{\mu \nu} q^{\nu}\right], \\
i \Gamma_{\mu}^{Z}\left(q^{2}\right)=i e\left[F_{L}^{Z}\left(q^{2}\right) \gamma_{\mu} P_{L}\right],
\end{gathered}
$$

where $q$ is the momentum of the $V$ boson. Actually, the most general Lorentz structure for on-shell fermions contains two additional (anapole) form factors, $F_{S}$ and $F_{P}$. 

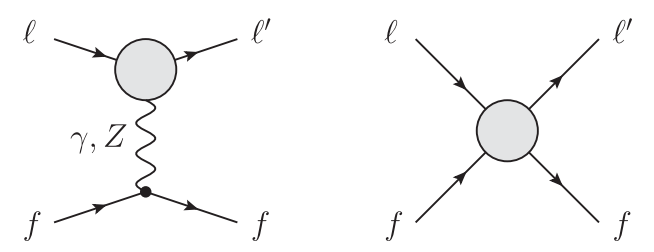

FIG. 1. Generic penguin and box diagrams contributing to $\ell \rightarrow$ $\ell^{\prime} \ell^{\prime \prime} \bar{\ell}^{\prime \prime \prime}\left(f=\ell^{\prime \prime}=\ell^{\prime \prime \prime}\right)$ and $\mu-e$ conversion in nuclei $(f=u, d)$.

However, they do not contribute when the $V$ boson is onshell, due to the transversality condition $q_{\mu} \epsilon^{\mu}=0$. The same happens for an off-shell $V$ boson when the masses of the external fermions can be neglected [73]. On the other hand, the dipole form factors (chirality flipping) are proportional to the external lepton masses.

In the limit $q^{2} \rightarrow 0$, appropriate for $\ell \rightarrow \ell^{\prime} \gamma$ and for the penguin contributions to $\ell \rightarrow \ell^{\prime} \ell^{\prime \prime} \bar{\ell}^{\prime \prime \prime}$ and $\mu-e$ conversion (Fig. 1), we may write:

$F_{L}^{\gamma}\left(q^{2}\right) \equiv q^{2} A_{1 L}, \quad F_{M}^{\gamma}\left(q^{2}\right) \simeq F_{M}^{\gamma}(0) \equiv \frac{m_{\ell}}{2} A_{2 R}$.

The vector form factor $F_{L}^{\gamma}$ for an on-shell photon vanishes by current conservation due to the electromagnetic gauge invariance, and hence only the dipole form factor $F_{M}^{\gamma}$ contributes to $\ell \rightarrow \ell^{\prime} \gamma$. Then the amplitude reads:

$\mathcal{M}\left(\ell \rightarrow \ell^{\prime} \gamma\right)=\operatorname{iem}_{\ell} A_{2 R} \bar{u}\left(p_{\ell^{\prime}}\right) P_{R} \sigma^{\mu \nu} q_{\nu} u\left(p_{\ell}\right) \epsilon_{\mu}^{\gamma}(q)$,

where $\epsilon_{\mu}^{\gamma}$ is the photon polarization vector and we have neglected the mass of the lighter lepton. The partial decay width is given by

$$
\Gamma\left(\ell \rightarrow \ell^{\prime} \gamma\right)=\alpha m_{\ell}^{3}\left|F_{M}^{\gamma}(0)\right|^{2} .
$$

The $Z \rightarrow \bar{\ell} \ell^{\prime}$ decay proceeds through the $Z \ell \ell^{\prime}$ vertex with $q^{2}=M_{Z}^{2}$. Here we can take both external leptons as massless and ignore the corresponding dipole form factor $F_{M}^{Z}$, hence omitted in (21). The amplitude is then given by

$$
\mathcal{M}\left(Z \rightarrow \bar{\ell} \ell^{\prime}\right)=e F_{L}^{Z}\left(M_{Z}^{2}\right) \bar{u}\left(p_{\ell^{\prime}}\right) \gamma^{\mu} P_{L} v\left(p_{\ell}\right) \epsilon_{\mu}^{Z}(q),
$$

where $\epsilon_{\mu}^{Z}$ is the $Z$ polarization vector and the partial decay width is

TABLE II. Possible LFV $\ell \rightarrow \ell^{\prime} \ell^{\prime \prime} \bar{\ell}^{\prime \prime \prime}$ channels.

\begin{tabular}{lllll}
\hline \hline Type & Flavors & $\ell \rightarrow \ell^{\prime} \ell^{\prime \prime} \bar{\ell}^{\prime \prime \prime}$ & & \\
\hline 1 & $\ell \neq \ell^{\prime}=\ell^{\prime \prime}=\ell^{\prime \prime \prime}$ & $\mu \rightarrow e e \bar{e}$ & $\tau \rightarrow e e \bar{e}$ & $\tau \rightarrow \mu \mu \bar{\mu}$ \\
2 & $\ell \neq \ell^{\prime} \neq \ell^{\prime \prime}=\ell^{\prime \prime \prime}$ & & $\tau \rightarrow e \mu \bar{\mu}$ & $\tau \rightarrow \mu e \bar{e}$ \\
3 & $\ell \neq \ell^{\prime}=\ell^{\prime \prime} \neq \ell^{\prime \prime \prime}$ & & $\tau \rightarrow e e \bar{\mu}$ & $\tau \rightarrow \mu \mu \bar{e}$ \\
\hline \hline
\end{tabular}

$$
\Gamma\left(Z \rightarrow \bar{\ell} \ell^{\prime}\right)=\frac{\alpha}{3} M_{Z}\left|F_{L}^{Z}\left(M_{Z}^{2}\right)\right|^{2} .
$$

Regarding $\ell \rightarrow \ell^{\prime} \ell^{\prime \prime} \bar{\ell}^{\prime \prime \prime}$, we distinguish the three types of decays in Table II. Apart from the photon-penguin and $Z$-penguin diagrams containing the effective $V \ell \ell^{\prime}$ vertices, these decays involve box diagrams (Fig. 1):

$$
\mathcal{M}\left(\ell \rightarrow \ell^{\prime} \ell^{\prime \prime} \bar{\ell}^{\prime \prime \prime}\right)=\mathcal{M}_{\gamma}+\mathcal{M}_{Z}+\mathcal{M}_{B},
$$

where

$$
\begin{aligned}
\mathcal{M}_{\gamma}= & \frac{e^{2}}{q^{2}} \bar{u}\left(p_{\ell^{\prime}}\right)\left(q^{2} A_{1 L} \gamma_{\mu} P_{L}+i m_{\ell} A_{2 R} P_{R} \sigma_{\mu \nu} q^{\nu}\right) u\left(p_{\ell}\right) \\
& \times \bar{u}\left(p_{\ell^{\prime \prime}}\right) \gamma^{\mu} v\left(p_{\ell^{\prime \prime \prime}}\right)-\left(\ell^{\prime} \leftrightarrow \ell^{\prime \prime}\right),
\end{aligned}
$$

$$
\begin{aligned}
\mathcal{M}_{Z}= & -\frac{e^{2}}{M_{Z}^{2}} F_{L}^{Z}(0) \bar{u}\left(p_{\ell^{\prime}}\right) \gamma_{\mu} P_{L} u\left(p_{\ell}\right) \\
& \times \bar{u}\left(p_{\ell^{\prime \prime}}\right) \gamma^{\mu}\left(g_{L}^{Z} P_{L}+g_{R}^{Z} P_{R}\right) v\left(p_{\ell^{\prime \prime \prime}}\right)-\left(\ell^{\prime} \leftrightarrow \ell^{\prime \prime}\right),
\end{aligned}
$$

$\mathcal{M}_{B}=e^{2} F_{B} \bar{u}\left(p_{\ell^{\prime}}\right) \gamma_{\mu} P_{L} u\left(p_{\ell}\right) \bar{u}\left(p_{\ell^{\prime \prime}}\right) \gamma^{\mu} P_{L} v\left(p_{\ell^{\prime \prime \prime}}\right)$,

with $g_{L, R}^{Z}$ the charged lepton couplings to the $Z$ boson in units of $e$,

$$
g_{L}^{Z}=\frac{1}{2 s_{W} c_{W}}\left(-1+2 s_{W}^{2}\right), \quad g_{R}^{Z}=\frac{s_{W}}{c_{W}},
$$

and the box diagrams are evaluated in the limit of zero external momenta. Channels of type 3 receive only box contributions, as they require two flavor-changing vertices. We have written the vector form factor $F_{L}^{\gamma}$ in terms of $A_{1 L}$ (22) to emphasize that the photon propagator cancels the $q^{2}$ prefactor. However, the dipole form factor $F_{M}^{\gamma}$, written here in terms of $A_{2 R}$ (22), will introduce a logarithmic dependence with the external lepton masses (they cannot be neglected) after phase-space integration of the squared amplitude. Notice that crossed diagrams with $\ell^{\prime}$ and $\ell^{\prime \prime}$ exchanged must be added, except for channels of type 2. In the box amplitude, the form factor $F_{B}$ includes the crossed contribution thanks to a Fierz identity [see Eq. (A4) of the Appendix A]. The expressions for the partial decay widths of $\ell \rightarrow \ell^{\prime} \ell^{\prime \prime} \bar{\ell}^{\prime \prime \prime}$ as a function of $A_{1 L}, A_{2 R}, F_{L}^{Z}(0)$, and $F_{B}$ are given in Appendix B.

The $\mu-e$ conversion in nuclei follows from similar diagrams as $\ell \rightarrow \ell^{\prime} \ell^{\prime \prime} \bar{\ell}^{\prime \prime \prime}$ replacing the last two leptons by a quark $q=u$ or $d$ (Fig. 1). It involves the same photonpenguin and $Z$-penguin and a couple of new box form factors, $F_{B_{u}}, F_{B_{d}}$ :

$$
\mathcal{M}_{B}^{q}=e^{2} F_{B_{q}} \bar{u}\left(p_{\mu}\right) \gamma_{\mu} P_{L} u\left(p_{q}\right) \bar{u}\left(p_{e}\right) \gamma^{\mu} P_{L} u\left(p_{q}^{\prime}\right) .
$$



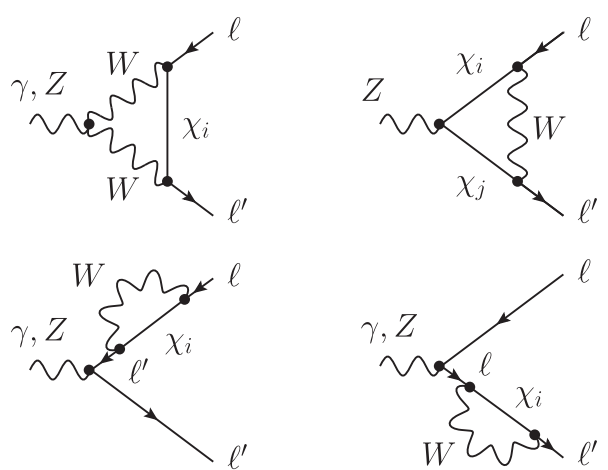

FIG. 2. One-loop diagrams contributing to the $V \ell \ell^{\prime}$ vertex. We have omitted here and elsewhere diagrams with would-beGoldstone fields, needed in the Feynman-'t Hooft gauge.

The expressions for the $\mu-e$ conversion rate in nuclei as a function of $A_{1 L}, A_{2 R}, F_{L}^{Z}(0), F_{B_{u}}$, and $F_{B_{d}}$ are given in Appendix C.

We have calculated in our model the one-loop contributions to the form factors introduced above, in the Feynman-'t Hooft gauge and using dimensional regularization. The effective LFV $V \ell \ell^{\prime}$ vertex is obtained from the diagrams of Fig. 2 supplemented by similar ones with the $W^{ \pm}$fields replaced by the would-be-Goldstone fields $G^{ \pm}$. The resulting photon form factors in the low $q^{2}$ limit are

$$
\begin{aligned}
& F_{L}^{\gamma}\left(q^{2}\right)=\frac{\alpha_{W}}{8 \pi M_{W}^{2}} \sum_{i}^{5} B_{\ell i}^{*} B_{\ell^{\prime} i} f_{L}^{\gamma}\left(x_{i} ; q^{2}\right), \\
& F_{M}^{\gamma}(0)=\frac{\alpha_{W}}{8 \pi M_{W}^{2}} \frac{m_{\ell}}{2} \sum_{i}^{5} B_{\ell i}^{*} B_{\ell^{\prime} i} f_{M}^{\gamma}\left(x_{i}\right),
\end{aligned}
$$

where $\alpha_{W}=\alpha / s_{W}^{2}, x_{i} \equiv m_{\chi_{i}}^{2} / M_{W}^{2}$ and

$$
\begin{aligned}
& f_{L}^{\gamma}\left(x ; q^{2}\right)= {\left[\frac{x^{2}\left(x^{2}-10 x+12\right) \ln x}{6(x-1)^{4}}\right.} \\
&\left.+\frac{\left(7 x^{3}-x^{2}-12 x\right)}{12(x-1)^{3}}-\frac{5}{9}\right] q^{2}+2 M_{W}^{2} \Delta_{\epsilon}, \\
& f_{M}^{\gamma}(x)=\frac{3 x^{3} \ln x}{2(x-1)^{4}}-\frac{2 x^{3}+5 x^{2}-x}{4(x-1)^{3}}+\frac{5}{6} .
\end{aligned}
$$

The term $\Delta_{\epsilon}=2 / \epsilon-\gamma_{E}+\ln 4 \pi+\ln \left(\mu^{2} / M_{W}^{2}\right)$ regulates the ultraviolet divergence in $4-\epsilon$ dimensions and cancels in (33) for $\ell \neq \ell^{\prime}$ due to the properties of $B$ (18). As expected, $F_{L}^{\gamma}(0)$ is zero.

From (34) one may derive the contribution $\delta a$ of heavy neutrinos to the muon dipole moment anomaly, $(g-2) / 2$. Subtracting that of light (massless) neutrinos, it reads: $\delta a=\frac{\alpha_{W}}{4 \pi} \frac{m_{\mu}^{2}}{M_{W}^{2}} \sum_{i=4}^{5}\left|B_{\mu i}\right|^{2}\left[\frac{3 x_{i}^{3} \ln x_{i}}{2\left(x_{i}-1\right)^{4}}-\frac{2 x_{i}^{3}+5 x_{i}^{2}-x_{i}}{4\left(x_{i}-1\right)^{3}}\right]$

that is negative, enhancing the disagreement with the current experimental measurement [64], but anyway negligible because the prefactor is $\sim 4 \times 10^{-11},\left|B_{\mu N_{i}}\right|^{2} \leq s_{\nu_{\mu}}^{2} \lesssim$ $10^{-3}$ and the absolute value of the remaining function is smaller than 0.5 .

Regarding the $F_{L}^{Z}$ form factor of the effective $Z \ell \ell^{\prime}$ vertex we find

$$
\begin{aligned}
F_{L}^{Z}\left(q^{2}\right)= & \frac{\alpha_{W}}{8 \pi s_{W} c_{W}} \sum_{i, j}^{5} B_{\ell i}^{*} B_{\ell^{\prime} j}\left[\delta_{i j} F\left(x_{i} ; q^{2}\right)\right. \\
& \left.+C_{i j}^{*} G\left(x_{i}, x_{j} ; q^{2}\right)+C_{i j} \sqrt{x_{i} x_{j}} H\left(x_{i}, x_{j} ; q^{2}\right)\right],
\end{aligned}
$$

where

$$
\begin{aligned}
& F\left(x ; q^{2}\right)= 2 c_{W}^{2}\left[q^{2}\left(\bar{C}_{1}+\bar{C}_{2}+\bar{C}_{12}\right)-6 \bar{C}_{00}+1\right] \\
&-\left(1-2 s_{W}^{2}\right) x \bar{C}_{00}-2 s_{W}^{2} x M_{W}^{2} \bar{C}_{0} \\
&+\frac{1}{2}\left(1-2 c_{W}^{2}\right)\left[(2+x) \bar{B}_{1}+1\right], \\
& G\left(x, y ; q^{2}\right)=-q^{2}\left(C_{0}+C_{1}+C_{2}+C_{12}\right)+2 C_{00}-1 \\
&-\frac{1}{2} x y M_{W}^{2} C_{0}, \\
& H\left(x, y ; q^{2}\right)=M_{W}^{2} C_{0}+\frac{1}{2} q^{2} C_{12}-C_{00}+\frac{1}{4},
\end{aligned}
$$

in full agreement with [19]. Here we have used the following shorthand notation for the standard PassarinoVeltman loop functions [74],

$C_{\{0,1,2,12.00\}}(x, y)=C_{\{0,1,2,12,00\}}\left(0, q^{2}, 0 ; M_{W}^{2}, x M_{W}^{2}, y M_{W}^{2}\right)$,

$\bar{C}_{\{0,1,2,12,00\}}(x)=C_{\{0,1,2,12,00\}}\left(0, q^{2}, 0 ; x M_{W}^{2}, M_{W}^{2}, M_{W}^{2}\right)$,

$$
\bar{B}_{1}(x)=B_{1}\left(0 ; x M_{W}^{2}, M_{W}^{2}\right),
$$

defined with the same conventions as the computer packages LoopTools [75] and COLLIER [76], that we have employed for numerical evaluations. Analytic expressions for these functions in the low $q^{2}$ limit, appropriate for the $Z$ penguin contribution to $\ell \rightarrow \ell^{\prime} \ell^{\prime \prime} \bar{\ell}^{\prime \prime \prime}$, can be found in [72] and have been cross-checked with the help of PACKAGE-X [77]. They are

$F(x ; 0)=\frac{5 x^{2} \ln x}{2(x-1)^{2}}-\frac{5 x}{2(x-1)}+\frac{1}{4}-\left(\frac{5}{2}-2 s_{W}^{2}\right) \Delta_{\epsilon}$, 

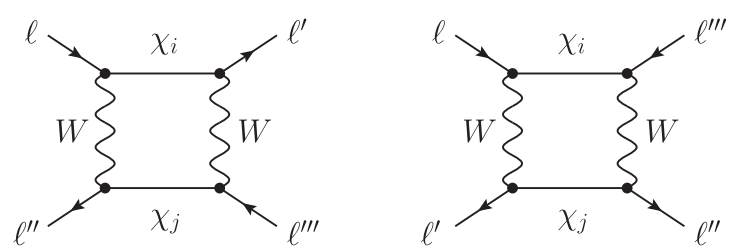

FIG. 3. Box diagrams contributing to $\ell \rightarrow \ell^{\prime} \ell^{\prime \prime} \bar{\ell}^{\prime \prime \prime}$. The diagram on the right introduces explicit LNV contributions.

$$
\begin{aligned}
G(x, y ; 0)= & \frac{1}{2(x-y)}\left[\frac{(y-1) x^{2} \ln x}{(x-1)}-\frac{(x-1) y^{2} \ln y}{(y-1)}\right] \\
& +\frac{1}{2}\left(\Delta_{\epsilon}-\frac{1}{2}\right), \\
H(x, y ; 0)= & \frac{1}{4(x-y)}\left[\frac{x(x-4) \ln x}{x-1}-\frac{y(y-4) \ln y}{y-1}\right] \\
& -\frac{1}{4}\left(\Delta_{\epsilon}+\frac{1}{2}\right) .
\end{aligned}
$$

The ultraviolet divergences cancel in (38) using the properties of the mixing matrices (18) and (19).

The box form factors are all finite. The amplitude for $\ell \rightarrow \ell^{\prime} \ell^{\prime \prime} \bar{\ell}^{\prime \prime \prime}$ receives the contribution of diagrams with explicit lepton number violating (LNV) vertices (Fig. 3). To implement the LNV vertices we have followed the algorithm in [78] that circumvents the explicit introduction of the charge conjugation matrix in the Feynman rules and allows to use Dirac propagators also for Majorana particles. In particular, the diagrams on the right of Fig. 3 contain genuine LNV contributions from Majorana particles that should vanish if lepton number is conserved. We have verified that this is indeed the case when the two fermion singlets form a Dirac field, i.e., when $\mu=0(r=1)$. The Lorentz structure of all box diagrams can be reduced to the form in (30) after some algebra (see Appendix A). In agreement with [72], we find

$$
\begin{aligned}
F_{B}= & \frac{\alpha_{W}}{16 \pi M_{W}^{2} s_{W}^{2}} \\
& \times\left\{\sum_{i, j}^{5}\left[B_{\ell i}^{*} B_{\ell^{\prime} i} B_{\ell^{\prime \prime \prime} j}^{*} B_{\ell^{\prime \prime} j}+\left(\ell^{\prime} \leftrightarrow \ell^{\prime \prime}\right)\right] f_{B_{d}}\left(x_{i}, x_{j}\right)\right. \\
& \left.+\sum_{i, j}^{5} B_{\ell i}^{*} B_{\ell^{\prime} j} B_{\ell^{\prime \prime \prime} i}^{*} B_{\ell^{\prime \prime} j} f_{B}^{\mathrm{LNV}}\left(x_{i}, x_{j}\right)\right\}
\end{aligned}
$$

where

$$
\begin{array}{r}
f_{B_{d}}(x, y)=\left(1+\frac{x y}{4}\right) \tilde{d}(x, y)-2 x y d(x, y), \\
f_{B}^{\mathrm{LNV}}(x, y)=\sqrt{x y}[2 \tilde{d}(x, y)-(4+x y) d(x, y)],
\end{array}
$$
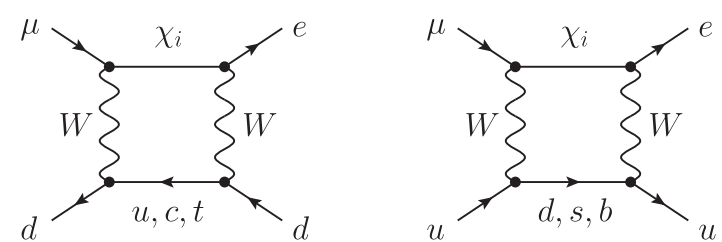

FIG. 4. Box diagrams contributing to $\mu-e$ conversion in nuclei.

and

$$
\begin{aligned}
\tilde{d}(x, y)= & \frac{x^{2} \ln x}{(1-x)^{2}(y-x)}+\frac{y^{2} \ln y}{(1-y)^{2}(x-y)} \\
& -\frac{1}{(1-x)(1-y)}, \\
d(x, y)= & \frac{x \ln x}{(1-x)^{2}(y-x)}+\frac{y \ln y}{(1-y)^{2}(x-y)} \\
& -\frac{1}{(1-x)(1-y)} .
\end{aligned}
$$

The one-loop contributions to the box form factors of $\mu-e$ conversion come from the diagrams in Fig. 4. We obtain:

$F_{B_{d}}=\frac{\alpha_{W}}{16 \pi m_{W}^{2} s_{W}^{2}} \sum_{i}^{5} \sum_{j}^{3} B_{\mu i}^{*} B_{e i}\left|V_{j d}\right|^{2} f_{B_{d}}\left(x_{i}, x_{j}^{u}\right)$,

$F_{B_{u}}=\frac{\alpha_{W}}{16 \pi m_{W}^{2} s_{W}^{2}} \sum_{i}^{5} \sum_{j}^{3} B_{\mu i}^{*} B_{e i}\left|V_{u j}\right|^{2} f_{B_{u}}\left(x_{i}, x_{j}^{d}\right)$,

where $x_{i}^{q}=m_{q_{i}}^{2} / M_{W}^{2}, V_{i j}$ is the CKM matrix and

$$
f_{B_{u}}(x, y)=-\left(4+\frac{x y}{4}\right) \tilde{d}(x, y)+2 x y d(x, y) .
$$

Neglecting all quark masses, except that of the top quark, and defining $x_{t}=m_{t}^{2} / m_{W}^{2}$, we may write:

$$
\begin{gathered}
\sum_{j}^{3}\left|V_{j d}\right|^{2} f_{B_{d}}\left(x_{i}, x_{j}^{u}\right) \\
=\left|V_{t d}\right|^{2}\left[f_{B_{d}}\left(x_{i}, x_{t}\right)-f_{B_{d}}\left(x_{i}, 0\right)\right]-f_{B_{d}}\left(x_{i}, 0\right), \\
\sum_{j}^{3}\left|V_{u j}\right|^{2} f_{B_{u}}\left(x_{i}, x_{j}^{d}\right)=f_{B_{u}}\left(x_{i}, 0\right) .
\end{gathered}
$$

In Appendix D we show how to express all these form factors in terms of the contributions of heavy neutrinos only. 


\section{NUMERICAL RESULTS}

Next we analyze the predictions of our model for different values of its free parameters: the three heavy-light mixings $s_{\nu_{e}}, s_{\nu_{\mu}}$ and $s_{\nu_{\tau}}$, the mass $m_{N_{1}}$ of the lightest heavy neutrino and the mass ratio $r=m_{N_{2}}^{2} / m_{N_{1}}^{2}$. If $r=1$ the two heavy Majorana neutrinos become a single Dirac field.

In order to be consistent with perturbative unitarity, the Yukawa couplings cannot exceed an upper limit. We will take

$$
Y_{\nu_{i}}=\frac{\sqrt{2 m_{N_{1}} m_{N_{2}}}}{v} s_{\nu_{i}}=\frac{\sqrt{2}}{v} m_{N_{1}} r^{1 / 4} s_{\nu_{i}}<\sqrt{4 \pi} .
$$

This means that, given $m_{N_{1}}$ and $r$,

$$
s_{\nu_{i}}<\frac{\sqrt{2 \pi} v}{m_{N_{1}} r^{1 / 4}},
$$

that constrains the mixings if $m_{N_{1}} r^{1 / 4} \gtrsim 620 \mathrm{GeV}$. In particular, $s_{\nu_{i}}<0.12$ for $m_{N_{1}} r^{1 / 4}=5 \mathrm{TeV}$. Given the mixings $s_{\nu_{i}}$ this condition also implies

$$
m_{N_{1}} r^{1 / 4}<\frac{\sqrt{2 \pi} v}{\max \left\{s_{\nu_{i}}\right\}}
$$

On the other hand, the heavy-light mixings must respect indirect constraints. We take $2 \sigma$ limits from the global fit to electroweak precision observables and lepton flavor conserving processes in [79], where the effects of extra neutrinos are encoded in effective operators ${ }^{2}$ :

$$
s_{\nu_{e}}<0.050, \quad s_{\nu_{\mu}}<0.021, \quad s_{\nu_{\tau}}<0.075 .
$$

Then (60) implies that $m_{N_{1}} r^{1 / 4}<8.2 \mathrm{TeV}$ if all mixings are fixed to the upper limits, but it could be larger otherwise.

\section{A. $\mu-e$ transitions}

LFV processes involving only $\mu-e$ transitions further constrain the masses and mixings of the heavy neutrinos in our model. Let us first consider the case of one singlet Dirac neutrino $\left(m_{N_{1}}=m_{N_{2}}=m_{N}\right)$. Figure 5 shows the contours in the $s_{\nu_{e}}-s_{\nu_{\mu}}$ plane that saturate present experimental bounds (solid lines) and the future sensitivities in Table I (dashed lines) for $m_{N}=0.1,5 \mathrm{TeV}$. In general the amplitudes for $Z \rightarrow \bar{\mu} e, \mu \rightarrow e e \bar{e}$ and $\mu-e$ conversion in nuclei depend on all three heavy-light mixing angles through the $Z$-penguin contribution, that involves the $C_{i j}$ matrix elements (17). Here we have assumed $s_{\nu_{\tau}}=0$, so the regions

\footnotetext{
${ }^{2}$ In a recent work [80] a global fit to modified neutrino couplings has been performed that alleviates the Cabibbo-angle anomaly and is compatible with the bounds we use.
}
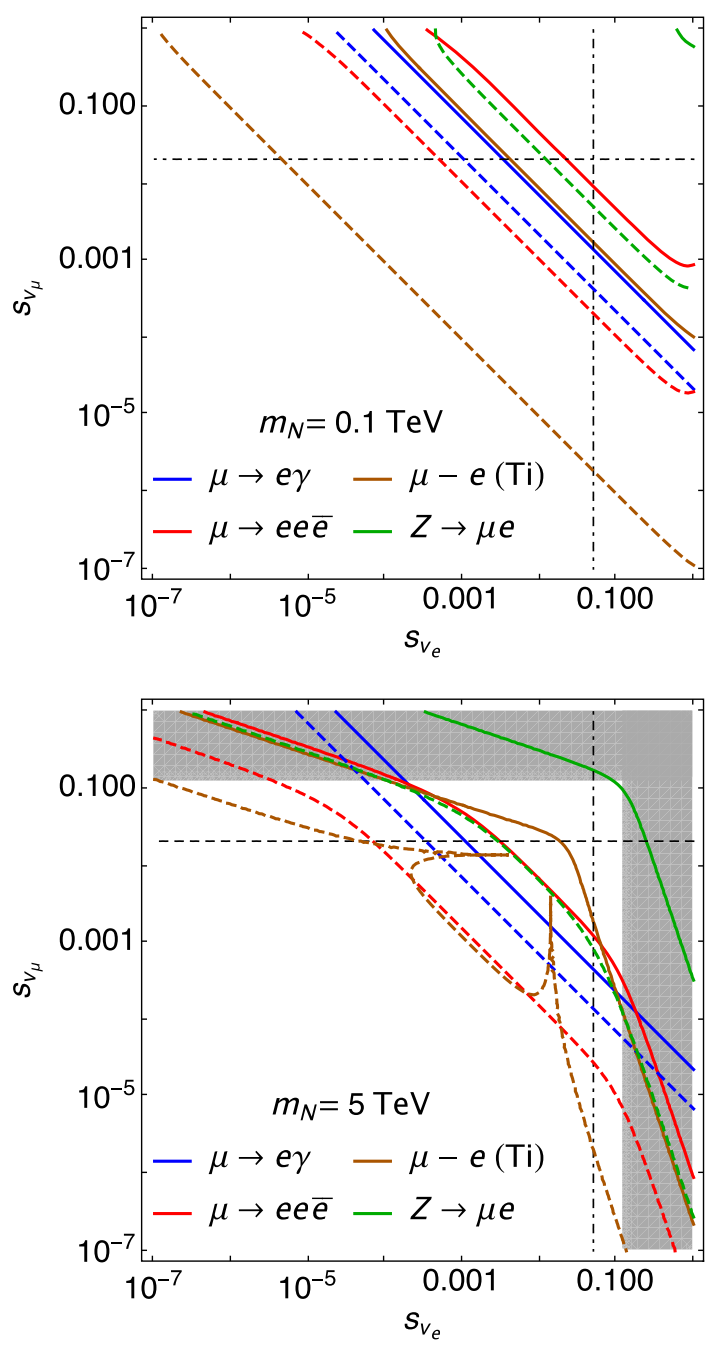

FIG. 5. Contour plots in the $s_{\nu_{e}}-s_{\nu_{\mu}}$ plane assuming degenerate heavy neutrino masses $m_{N_{1}}=m_{N_{2}}=m_{N}=0.1,5 \mathrm{TeV}$ that saturate present limits (solid lines) and future sensitivities (dashed lines) of several $\mu-e$ transitions. A conservative value $s_{\nu_{\tau}}=0$ is assumed when needed. The black dot-dashed lines show current indirect limits. The gray-shadowed region is beyond the perturbative limit $Y_{\nu_{i}}^{2}<4 \pi$.

below the curves in Fig. 5 enclose the most conservative values for $s_{\nu_{e}}$ and $s_{\nu_{\mu}}$ (i.e., a nonzero $s_{\nu_{\tau}}$ would imply stronger bounds). Actually, $\mu \rightarrow$ e $\gamma$ sets stringent constrains only on the product $s_{\nu_{e}} s_{\nu_{\mu}}$; for $m_{N} \gtrsim 1 \mathrm{TeV}$ we find that this process does not depend on the heavy neutrino masses and that its branching fraction can be approximated by

$$
\mathrm{BR}(\mu \rightarrow e \gamma) \approx \frac{3 \alpha}{8 \pi} s_{\nu_{e}}^{2} s_{\nu_{\mu}}^{2}
$$

which yields the conservative direct limit:

$$
s_{\nu_{e}}^{2} s_{\nu_{\mu}}^{2}<5.1 \times 10^{-10} .
$$


Figure 5 also reveals that in forthcoming experiments $\mu-e$ (Ti) and $\mu \rightarrow e e \bar{e}$ will be more constraining than $\mu \rightarrow e \gamma$.

Another point that we would like to emphasize is that the amplitudes for $\mu \rightarrow e e \bar{e}, \mu-e$ conversion in nuclei and $Z \rightarrow \mu e$ introduce terms of order $s_{\nu_{i}}^{4}$ that cannot be ignored, since they imply a strong quadratic dependence on the heavy neutrino masses. Indeed, these terms dominate the amplitude when the splitting between the two heavy masses is large. Our results differ then from those in [79], where as a first approximation the terms proportional to $s_{\nu_{i}}^{4}$ are neglected.

From a phenomenological point of view it is also interesting to investigate whether the model can accommodate values of the different observables involving $\mu-e$ transitions near the current experimental bounds. What are the maximum values of $\mu \rightarrow e \gamma, \mu \rightarrow e e \bar{e}, \mu-e$ (Ti) and $Z \rightarrow \mu e$ consistent with all the bounds in heavy neutrino models? To answer this question we have considered the cases with low $(r=1)$ and high $(r=25)$ neutrino mass ratio and different values of $s_{\nu_{e}} s_{\nu_{\mu}}, m_{N}$, and $s_{\nu_{\tau}}$ that respect the indirect and perturbative limits.

In Fig. 6 we plot the ratio of the different observables to their current bound. In general, it is $\mu \rightarrow e \gamma$ the most constraining process, so in the plot we set the maximum value of $s_{\nu_{e}} s_{\nu_{\mu}}$ compatible with that process and vary the rest of the parameters. The lower (upper) curves of each band correspond to $s_{\nu_{\tau}}=0\left(s_{\nu_{\tau} \max }=0.075\right)$, whereas the drop in the $\mu-e$ conversion amplitude at neutrino masses $\lesssim 2 \mathrm{TeV}$ is due to the opposite sign in the form factors $F_{B_{d}}$ and $F_{B_{u}}$.

We find that $\mu-e$ (Ti) may also saturate its present bounds if the neutrino masses are large enough: $m_{N}>$ $5.5 \mathrm{TeV}$ for a Dirac neutrino $(r=1)$ and $m_{N}>4.7 \mathrm{TeV}$ if $r=25$. In contrast, in these models the processes $\mu \rightarrow e e \bar{e}$ and $Z \rightarrow \mu e$ cannot reach their current experimental limits consistently with $\mu \rightarrow e \gamma$ and $\mu-e$ (Ti) for any values of the free parameters. We find

$$
\mathrm{BR}(\mu \rightarrow e e \bar{e})<5.2 \times 10^{-13},
$$

and

$$
\mathrm{BR}(Z \rightarrow \mu e)<6.5 \times 10^{-13} .
$$

These limits do not change for lower values of $s_{\nu_{e}} s_{\nu_{\mu}}$, so our result implies that the observation at future experiments of any of these processes at a rate between the current bounds and these upper limits would exclude heavy neutrinos (both Dirac or Majorana) as a possible explanation. Larger values of the Majorana mass ratio $r$ than the one shown in the second plot of Fig. 6 would result in similar allowed regions just cutting off the higher masses to meet the perturbative unitarity limit.
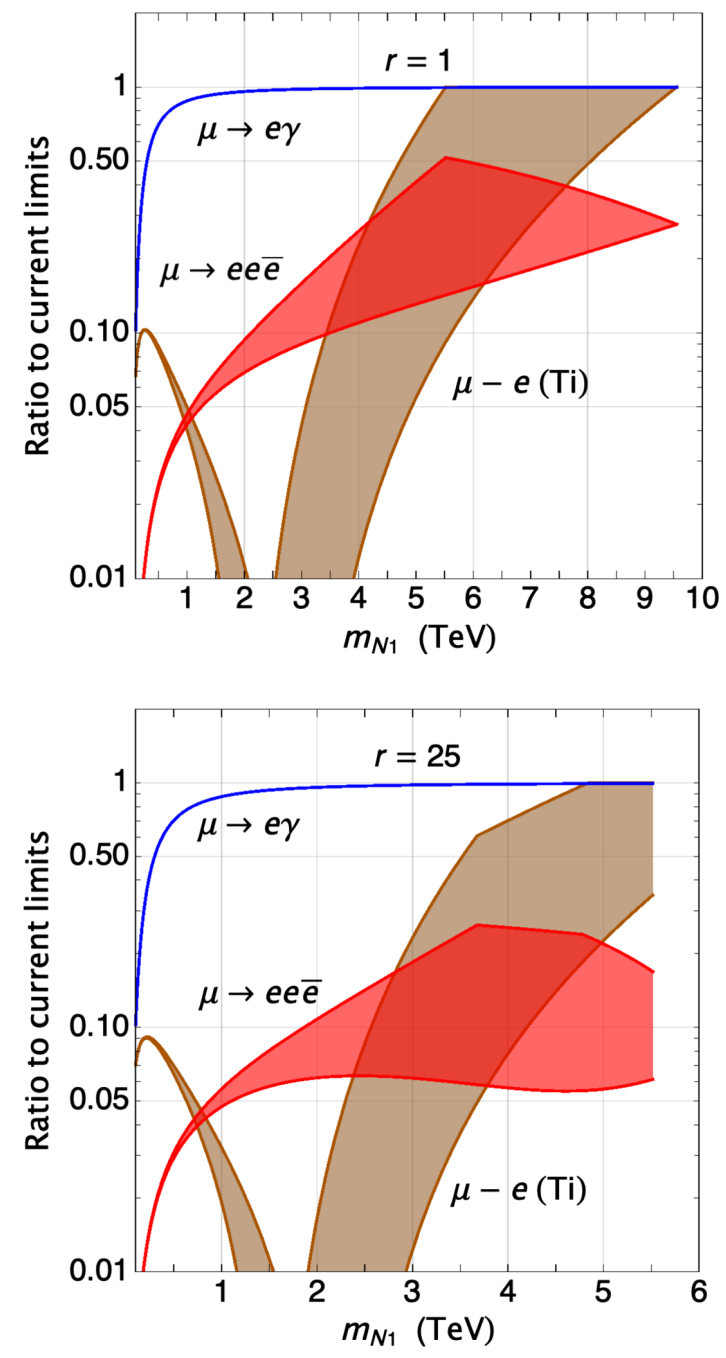

FIG. 6. Predictions normalized to current limits assuming fixed maximum mixings $s_{\nu_{e}}^{\max }$ and $s_{\nu_{\mu}}^{\max }$ compatible with $\mu \rightarrow e \gamma$ (63) and the indirect limits (61), for two values of the neutrino mass-ratio $r$. The predictions for $\mu \rightarrow e e \bar{e}$ and $\mu-e$ (Ti) get constrained to the corresponding bands whose upper (lower) boundaries are determined by $s_{\nu_{\tau}}=0\left(s_{\nu_{\tau}}^{\max }\right)$. Higher masses are forbidden by perturbative unitarity (60) for these mixings. The ratio of $\operatorname{BR}(Z \rightarrow \mu e)$ to current limits is always much smaller than the others, below $10^{-6}$.

Looking at the improvement factor of the sensitivities in future experiments (Table I) we conclude that $\mu-e$ (Ti) will take the lead in constraining the parameter space of our model, rather than $\mu \rightarrow e \gamma$, except for a tiny region of masses between 1.5 and $2 \mathrm{TeV}$ that would be probed better by $\mu \rightarrow e e \bar{e}$.

\section{B. $\tau-e$ transitions}

The constraints on our model from current limits on LFV $\tau-e$ transitions (Table I) turn out to be less restrictive than those involving the first two lepton families. In Fig. 7 we show our predictions for the maximum possible rates for 

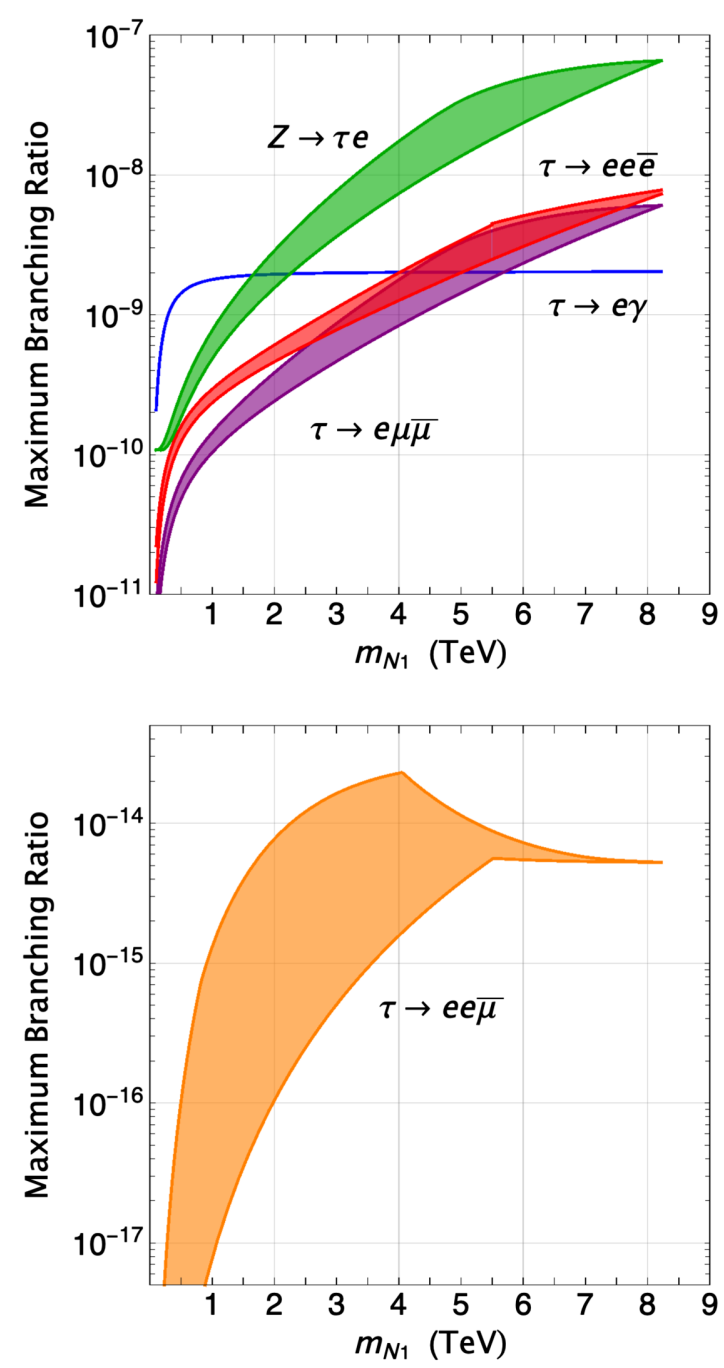

FIG. 7. Maximum values of $\tau-e$ transition rates compatible with current constraints on $\mu \rightarrow e \gamma, \mu-e$ (Ti) and the indirect limits (61). These maximum rates get constrained to the corresponding bands whose lower (upper) boundaries are determined by $r=1(r \gg 1)$.

several LFV $\tau$ decays consistent with current bounds from $\mu \rightarrow e \gamma$ and $\mu-e$ (Ti), the indirect limits (61) and perturbative unitarity (60).

The maximum branching ratio for $\tau \rightarrow e \gamma$, independent of heavy neutrino masses as for $\mu \rightarrow e \gamma$, is

$$
\mathrm{BR}(\tau \rightarrow e \gamma)<2.0 \times 10^{-9}
$$

close but still below the future sensitivity of Belle-II.

The predictions for $\tau \rightarrow e e \bar{e}$ and $\tau \rightarrow e \mu \bar{\mu}$ are very similar because the dominant contribution comes in both cases from the $Z$ penguin diagram. They can reach:

$$
\operatorname{BR}(\tau \rightarrow e e \bar{e})<7.3 \times 10^{-9},
$$

$$
\operatorname{BR}(\tau \rightarrow e \mu \bar{\mu})<6.0 \times 10^{-9},
$$

which, unlike $\tau \rightarrow e \gamma$, are well within the expected sensitivity of Belle-II. These maximum values correspond to a Dirac neutino singlet $(r=1)$ with a mass just below the perturbative limit for the largest posible mixings, $m_{N}=8.2 \mathrm{TeV}$. For smaller masses there is room for $r>1$ that enhance the decay rates up to the upper part of the shaded band in Fig 7.

The decay $\tau \rightarrow e e \bar{\mu}$ (double flavor change) is generated through box diagrams only, so its amplitude is always proportional to $s_{\nu_{e}}^{2} s_{\nu_{\tau}} s_{\nu_{\mu}}$ and hence more suppressed than the other channels (see lower plot of Fig. 7), with a maximum at

$$
\mathrm{BR}(\tau \rightarrow e e \bar{\mu})<2.3 \times 10^{-14} .
$$

Nevertheless, it is important to remark that this decay is sensitive to the genuine effects of Majorana neutrinos encoded in the LNV vertices of one of its box contributions. Unlike the other processes, the rate of this for two nondegenerate Majorana neutrinos can be enhanced by more than two orders of magnitude when compared to the case of a Dirac singlet. In fact, the maximum branching ratio above is obtained for $r \approx 16.9$ and $m_{N_{1}} \approx 4.1 \mathrm{TeV}$.

For $Z \rightarrow \tau e$ our model predicts

$$
\mathrm{BR}(Z \rightarrow \tau e)<6.0 \times 10^{-8},
$$

which is at the reach of future circular colliders.

Concerning the maximum values for the rates of $\tau-\mu$ transitions, we get similar results as above by exchanging $e$ and $\mu$ and applying some approximate correction factors. In particular, taking the maximal possible mixings from the indirect limits (61), the processes $\tau \rightarrow \mu \gamma, \tau \rightarrow \mu \mu \bar{\mu}$, $\tau \rightarrow \mu e \bar{e}$, and $Z \rightarrow \tau \mu$ are suppressed by a factor of $\left(s_{\nu_{\mu}}^{\max } / s_{\nu_{e}}^{\max }\right)^{2} \approx 0.18$, whereas $\tau \rightarrow \mu \mu \bar{e}$ is enhanced by $s_{\nu_{e}}^{\max } / s_{\nu_{\mu}}^{\max } \approx 2.4$.

\section{CONCLUSIONS}

The lepton sector of the SM is still poorly known. In particular, we do not know whether the observed neutrinos are Dirac or Majorana particles or if the sector includes additional fermion singlets (sterile neutrinos). These extra neutrinos, if any, would enhance cLFV processes that are otherwise very suppressed by the tiny masses of the observed neutrinos.

In this work we have introduced the simplest neutrino model that captures all the effects that are relevant to these processes: a minimal number of Majorana neutrino fields (three active and two sterile), two of them heavy and the rest massless, allowing unsuppressed heavy-light mixings 
and the possibility of LNV encoded in the mass splitting of the heavy states $\left(\mu=m_{N_{2}}-m_{N_{1}}=(\sqrt{r}-1) m_{N_{1}}\right)$. Larger splittings, however, imply an increasing amount of fine tuning required to cancel loop corrections that deform the proposed pattern, which is only stable in the lepton number conserving case with $\mu=0$. In any case, only five parameters describe the model, that are expressed in terms of the two heavy masses and the three heavy-light mixings $\left(s_{\nu_{k}}^{2}, k=e, \mu, \tau\right)$. The model could be perturbed to account for the extremely light neutrino masses $\left(m_{\nu_{i}}<1 \mathrm{eV}\right)$ and the observed Pontecorvo-Maki-Nakagawa-Sakata (PMNS) mixings, but this would have no impact on cLFV.

We have explored the predictions of our model for the most relevant reactions involving one or two flavor changes. We have presented analytical expressions for all of them and calculated their expected rates compatible with present direct and indirect limits. Our computation is exact at one loop, including all orders in the heavy-light mixings, and the genuine Majorana effects have been singled out. This work completes and updates previous results and is in agreement with an effective field theory analysis of the most general seesaw extension of the SM [81]. We conclude that forthcoming LFV experiments will probe a significant fraction of the parameter space of models with heavy Majorana neutrinos.

\section{ACKNOWLEDGMENTS}

We would like to thank F. del Águila and José Santiago for helpful discussions. This work was supported in part by the Spanish Ministry of Science, Innovation and Universities, under Grant No. FPA2016-78220-C3-1,2,3$\mathrm{P}$ (fondos FEDER), and Junta de Andalucía, Grants No. FQM 101 and No. SOMM17/6104/UGR. G. H. T. wants to acknowledge financial support from Conacyt through the program "Estancia Postdoctoral en el Extranjero." The work of P. R. has been partially funded by Conacyt through the Project No. 250628 (Ciencia Básica) and Fondo SEP-Cinvestav 2018 (Project No. 142).

\section{APPENDIX A: USEFUL IDENTITIES}

In the limit of zero external momenta the Lorentz structure of all box diagrams can be reduced to the same form using several identities based on the decomposition in the chiral basis of Dirac matrices, transpositions and Fierz rearrangements:

$$
\begin{gathered}
\bar{u}\left(p_{1}\right) \gamma^{\mu} \gamma^{\alpha} \gamma^{\nu} P_{L} u(p) \bar{u}\left(p_{2}\right) \gamma_{\nu} \gamma_{\alpha} \gamma_{\mu} P_{L} v\left(p_{3}\right) \\
=4 \bar{u}\left(p_{1}\right) \gamma^{\mu} P_{L} u(p) \bar{u}\left(p_{2}\right) \gamma_{\mu} P_{L} v\left(p_{3}\right), \\
\bar{u}\left(p_{1}\right) \gamma^{\mu} \gamma^{\alpha} \gamma^{\nu} P_{L} u(p) \bar{u}\left(p_{2}\right) \gamma_{\mu} \gamma_{\alpha} \gamma_{\nu} P_{L} v\left(p_{3}\right) \\
=16 \bar{u}\left(p_{1}\right) \gamma^{\mu} P_{L} u(p) \bar{u}\left(p_{2}\right) \gamma_{\mu} P_{L} v\left(p_{3}\right),
\end{gathered}
$$

$$
\begin{gathered}
\bar{u}\left(p_{3}\right) \gamma^{\mu} \gamma^{\nu} P_{L} u(p) \bar{u}\left(p_{2}\right) \gamma_{\mu} \gamma_{\nu} P_{L} v\left(p_{1}\right) \\
=4 \bar{u}\left(p_{3}\right) P_{L} u(p) \bar{u}\left(p_{2}\right) P_{R} v\left(p_{1}\right) \\
=-4 \bar{v}(p) P_{L} v\left(p_{3}\right) \bar{u}\left(p_{2}\right) P_{R} v\left(p_{1}\right) \\
=-\frac{1}{2} \bar{v}(p) \gamma^{\mu} P_{R} v\left(p_{1}\right) \bar{u}\left(p_{2}\right) \gamma_{\mu} P_{L} v\left(p_{3}\right) \\
=-\frac{1}{2} \bar{u}\left(p_{1}\right) \gamma^{\mu} P_{L} u(p) \bar{u}\left(p_{2}\right) \gamma_{\mu} P_{L} v\left(p_{3}\right), \\
\bar{u}\left(p_{2}\right) \gamma^{\mu} P_{L} u(p) \bar{u}\left(p_{1}\right) \gamma_{\mu} P_{L} v\left(p_{3}\right) \\
=-\bar{u}\left(p_{1}\right) \gamma^{\mu} P_{L} u(p) \bar{u}\left(p_{2}\right) \gamma_{\mu} P_{L} v\left(p_{3}\right) .
\end{gathered}
$$

\section{APPENDIX B: PARTIAL DECAY WIDTHS FOR LFV THREE-BODY DECAYS}

Given the generic form factors in Eqs. (27)-(30) the expressions for the partial widths of the three types of decays in Table II are [82]:

$$
\begin{aligned}
\Gamma_{1}= & \frac{\alpha^{2} m_{\ell}^{5}}{96 \pi}\left\{3\left|A_{1 L}\right|^{2}+2\left|A_{2 R}\right|^{2}\left(8 \ln \frac{m_{\ell}}{m_{\ell^{\prime \prime}}}-13\right)\right. \\
& +2\left|F_{L L}\right|^{2}+\left|F_{L R}\right|^{2}+\frac{1}{2}\left|F_{B}\right|^{2}-\left[6 A_{1 L} A_{2 R}^{*}-F_{L L} F_{B}^{*}\right. \\
& \left.\left.-\left(A_{1 L}-2 A_{2 R}\right)\left(2 F_{L L}^{*}+F_{L R}^{*}+F_{B}^{*}\right)+\text { H.c. }\right]\right\}, \quad(\mathrm{B} 1) \\
\Gamma_{2}= & \frac{\alpha^{2} m_{\ell}^{5}}{96 \pi}\left\{2\left|A_{1 L}\right|^{2}+4\left|A_{2 R}\right|^{2}\left(4 \ln \frac{m_{\ell}}{m_{\ell^{\prime \prime}}}-7\right)\right. \\
& +\left|F_{L L}\right|^{2}+\left|F_{L R}\right|^{2}+\left|F_{B}\right|^{2}-\left[4 A_{1 L} A_{2 R}^{*}-\frac{1}{2} F_{L L} F_{B}^{*}\right. \\
& \left.\left.-\left(A_{1 L}-2 A_{2 R}\right)\left(F_{L L}^{*}+F_{L R}^{*}+\frac{1}{2} F_{B}^{*}\right)+\text { H.c. }\right]\right\},
\end{aligned}
$$

$$
\Gamma_{3}=\frac{\alpha^{2} m_{\ell}^{5}}{192 \pi}\left|F_{B}\right|^{2}
$$

where

$$
F_{L L}=-\frac{F_{L}^{Z}(0) g_{L}^{Z}}{M_{Z}^{2}}, \quad F_{L R}=-\frac{F_{L}^{Z}(0) g_{R}^{Z}}{M_{Z}^{2}}
$$

and the $Z$ couplings $g_{L, R}^{Z}$ are given in Eq. (31).

\section{APPENDIX C: THE $\mu-e$ CONVERSION RATE}

In terms of the form factors in Eqs. (27), (28), (29), and (32), the $\mu-e$ conversion rate in a nucleus with $Z$ protons and $N=A-Z$ neutrons is given by 
TABLE III. Input parameters for different nuclei $[83,84]$.

\begin{tabular}{lrcccc}
\hline \hline Nucleus & $N$ & $Z$ & $Z_{\text {eff }}$ & $F_{P}$ & $\Gamma_{\text {capt }}[\mathrm{GeV}]$ \\
\hline${ }_{13}^{27} \mathrm{Al}$ & 14 & 13 & 11.5 & 0.64 & $4.6 \times 10^{-19}$ \\
${ }^{48} \mathrm{Ti}$ & 26 & 22 & 17.6 & 0.54 & $1.7 \times 10^{-18}$ \\
${ }_{197}^{197} \mathrm{Au}$ & 118 & 79 & 33.5 & 0.16 & $8.6 \times 10^{-18}$ \\
\hline \hline
\end{tabular}

$$
\begin{aligned}
\mathcal{R}= & \frac{\alpha^{5} Z_{\text {eff }}^{4}}{\Gamma_{\text {Capt }} Z} F_{P}^{2} m_{\mu}^{5} \mid 2 Z\left(A_{1 L}+A_{2 R}\right) \\
& -(2 Z+N)\left(F_{L L}^{u}+F_{L R}^{u}+B_{L}^{u}\right) \\
& -\left.(Z+2 N)\left(F_{L L}^{d}+F_{L R}^{d}+B_{L}^{d}\right)\right|^{2},
\end{aligned}
$$

where

$$
F_{L L}^{q}=-\frac{F_{L}^{Z}(0) g_{L q}^{Z}}{M_{Z}^{2}}, \quad F_{L R}^{q}=-\frac{F_{L}^{Z}(0) g_{R q}^{Z}}{M_{Z}^{2}},
$$

$$
\begin{array}{ll}
g_{L u}^{Z}=\frac{1-\frac{4}{3} s_{W}^{2}}{2 s_{W} c_{W}}, & g_{R u}^{Z}=-\frac{2 s_{W}}{3 c_{W}}, \\
g_{L d}^{Z}=\frac{-1+\frac{2}{3} s_{W}^{2}}{2 s_{W} c_{W}}, & g_{R d}^{Z}=\frac{s_{W}}{3 c_{W}},
\end{array}
$$

and the rest of the parameters are in Table III.

\section{APPENDIX D: EXPRESSIONS IN TERMS OF MASSIVE NEUTRINOS ONLY}

Using the relations between mixings matrices (18) and (19) with $\ell \neq \ell^{\prime}$, one may write the generic contributions to all form factors in terms of massive neutrinos only, $m_{N_{1}}=m_{\chi_{4}}, m_{N_{2}}=m_{\chi_{5}}$ :

$$
\sum_{i}^{5} B_{\ell i}^{*} B_{\ell^{\prime} i} f\left(x_{i}\right)=\sum_{i}^{2} B_{\ell N_{i}}^{*} B_{\ell^{\prime} N_{i}}\left[f\left(x_{N_{i}}\right)-f(0)\right]
$$

$$
\begin{aligned}
\sum_{i, j}^{5} B_{\ell i}^{*} B_{\ell^{\prime} i} B_{\ell^{\prime \prime \prime} j}^{*} B_{\ell^{\prime \prime} j} f\left(x_{i}, x_{j}\right)= & \sum_{i, j}^{2} B_{\ell N_{i}}^{*} B_{\ell^{\prime} N_{j}} \delta_{\ell^{\prime \prime} \ell^{\prime \prime \prime}} \delta_{N_{i} N_{j}}\left[f\left(x_{N_{i}}, 0\right)-f(0,0)\right]+\sum_{i, j}^{2} B_{\ell N_{i}}^{*} B_{\ell^{\prime} N_{i}} B_{\ell^{\prime \prime \prime} N_{j}}^{*} B_{\ell^{\prime \prime} N_{j}}\left[f\left(x_{N_{i}}, x_{N_{j}}\right)\right. \\
& \left.-f\left(x_{N_{i}}, 0\right)-f\left(0, x_{N_{i}}\right)+f(0,0)\right] .
\end{aligned}
$$

[1] S. L. Glashow, Nucl. Phys. 22, 579 (1961).

[2] S. Weinberg, Phys. Rev. Lett. 19, 1264 (1967).

[3] A. Salam, Conf. Proc. C 680519, 244 (1968).

[4] Y. Fukuda et al. (Super-Kamiokande Collaboration), Phys. Rev. Lett. 81, 1562 (1998).

[5] Q. R. Ahmad et al. (SNO Collaboration), Phys. Rev. Lett. 87, 071301 (2001).

[6] Q. R. Ahmad et al. (SNO Collaboration), Phys. Rev. Lett. 89, 011301 (2002).

[7] R. N. Mohapatra and P. B. Pal, World Sci. Lect. Notes Phys. 60, 1 (1998); 72, 1 (2004).

[8] B. Pontecorvo, Zh. Eksp. Teor. Fiz. 34, 247 (1957) [Sov. Phys. JETP 7, 172 (1958)].

[9] Z. Maki, M. Nakagawa, and S. Sakata, Prog. Theor. Phys. 28, 870 (1962).

[10] N. Cabibbo, Phys. Rev. Lett. 10, 531 (1963).
[11] M. Kobayashi and T. Maskawa, Prog. Theor. Phys. 49, 652 (1973).

[12] P. Minkowski, Phys. Lett. 67B, 421 (1977).

[13] M. Gell-Mann, P. Ramond, and R. Slansky, Conf. Proc. C 790927, 315 (1979).

[14] R. N. Mohapatra and G. Senjanovic, Phys. Rev. Lett. 44, 912 (1980).

[15] S. T. Petcov, Yad. Fiz. 25, 641 (1977) [Sov. J. Nucl. Phys. 25, 340 (1977)]; Yad. Fiz. 25, 1336(E) (1977) [Sov. J. Nucl. Phys. 25, 698(E) (1977)].

[16] S. M. Bilenky, S. T. Petcov, and B. Pontecorvo, Phys. Lett. 67B, 309 (1977).

[17] T. P. Cheng and L. F. Li, Gauge Theory Of Elementary Particle Physics (Clarendon, Oxford Science Publications, Oxford, 1984), p. 536.

[18] J. I. Illana, M. Jack, and T. Riemann, arXiv:hep-ph/0001273. 
[19] J. I. Illana and T. Riemann, Phys. Rev. D 63, 053004 (2001).

[20] G. Hernández-Tomé, G. L. Castro, and P. Roig, Eur. Phys. J. C 79, 84 (2019).

[21] P. Blackstone, M. Fael, and E. Passemar, arXiv:1912.09862.

[22] E. Arganda, A. M. Curiel, M. J. Herrero, and D. Temes, Phys. Rev. D 71, 035011 (2005).

[23] D. N. Dinh, A. Ibarra, E. Molinaro, and S. T. Petcov, J. High Energy Phys. 08 (2012) 125; 09 (2013) 23.

[24] D. N. Dinh and S. T. Petcov, J. High Energy Phys. 09 (2013) 086.

[25] A. Abada, V. De Romeri, S. Monteil, J. Orloff, and A. M. Teixeira, J. High Energy Phys. 04 (2015) 051.

[26] E. Arganda, M. J. Herrero, X. Marcano, and C. Weiland, Phys. Rev. D 91, 015001 (2015).

[27] V. De Romeri, M. J. Herrero, X. Marcano, and F. Scarcella, Phys. Rev. D 95, 075028 (2017).

[28] M. Lindner, M. Platscher, and F. S. Queiroz, Phys. Rep. 731, 1 (2018).

[29] R. N. Mohapatra and J. W. F. Valle, Phys. Rev. D 34, 1642 (1986).

[30] J. Bernabéu, A. Santamaría, J. Vidal, A. Méndez, and J. W. F. Valle, Phys. Lett. B 187, 303 (1987).

[31] M. Malinsky, J. C. Romao, and J. W. F. Valle, Phys. Rev. Lett. 95, 161801 (2005).

[32] F. del Aguila, M. Masip, and J. L. Padilla, Phys. Lett. B 627, 131 (2005).

[33] F. del Aguila, L. Ametller, J. I. Illana, J. Santiago, P. Talavera, and R. Vega-Morales, J. High Energy Phys. 08 (2017) 028; 02 (2019) 47.

[34] F. del Aguila, J. I. Illana, J. M. Perez-Poyatos, and J. Santiago, J. High Energy Phys. 12 (2019) 154.

[35] A. Abada, M. E. Krauss, W. Porod, F. Staub, A. Vicente, and C. Weiland, J. High Energy Phys. 11 (2014) 048.

[36] E. Arganda, M. J. Herrero, X. Marcano, and C. Weiland, Phys. Rev. D 93, 055010 (2016).

[37] E. Arganda, M. J. Herrero, X. Marcano, and C. Weiland, Phys. Lett. B 752, 46 (2016).

[38] N. Arkani-Hamed, S. Dimopoulos, and G. R. Dvali, Phys. Lett. B 429, 263 (1998).

[39] L. Randall and R. Sundrum, Phys. Rev. Lett. 83, 3370 (1999).

[40] L. Coito, C. Faubel, and A. Santamaria, arXiv:1912.10001 [Phys. Rev. D (to be published)].

[41] W. H. Furry, Phys. Rev. 56, 1184 (1939).

[42] Y. B. Zeldovich and M. Y. Khlopov, Pis'ma Zh. Eksp. Teor. Fiz. 34, 148 (1981).

[43] A. Ilakovac, B. A. Kniehl, and A. Pilaftsis, Phys. Rev. D 52, 3993 (1995).

[44] A. Ilakovac, Phys. Rev. D 54, 5653 (1996).

[45] V. Gribanov, S. Kovalenko, and I. Schmidt, Nucl. Phys. B607, 355 (2001).

[46] L. S. Littenberg and R. E. Shrock, Phys. Rev. D 46, R892 (1992).

[47] C. Barbero, G. L. Castro, and A. Mariano, Phys. Lett. B 566, 98 (2003).

[48] A. M. Baldini et al. (MEG II Collaboration), Eur. Phys. J. C 78, 380 (2018).

[49] A. Blondel et al., arXiv:1301.6113.
[50] A. Alekou et al., arXiv:1310.0804.

[51] Y. Kuno (COMET Collaboration), Prog. Theor. Exp. Phys. 2013, 022C01 (2013).

[52] E. Kou et al. (Belle-II Collaboration), Prog. Theor. Exp. Phys. 2019, 123C01 (2019).

[53] C. Hays, M. Mitra, M. Spannowsky, and P. Waite, J. High Energy Phys. 05 (2017) 014.

[54] R. Aaij et al. (LHCb Collaboration), J. High Energy Phys. 02 (2015) 121.

[55] M. Dam, SciPost Phys. Proc. 1, 041 (2019).

[56] A. Blondel et al., arXiv:1906.02693.

[57] J. B. G. da Costa et al. (CEPC Study Group), arXiv: 1811.10545.

[58] A. Cerri et al., Opportunities in Flavour Physics at the HLLHC and HE-LHC, CERN Yellow Reports: Monographs Vol. 7 (2019), pp. 867-1158.

[59] Y. Amhis et al. (HFLAV Collaboration), Eur. Phys. J. C 77, 895 (2017).

[60] L. Calibbi and G. Signorelli, Riv. Nuovo Cimento 41, 71 (2018).

[61] J. Adam et al. (MEG Collaboration), Phys. Rev. Lett. 110, 201801 (2013).

[62] W. H. Bertl et al. (SINDRUM II Collaboration), Eur. Phys. J. C 47, 337 (2006).

[63] B. Aubert et al. (BABAR Collaboration), Phys. Rev. Lett. 104, 021802 (2010).

[64] M. Tanabashi et al. (Particle Data Group), Phys. Rev. D 98, 030001 (2018).

[65] U. Bellgardt et al. (SINDRUM Collaboration), Nucl. Phys. B299, 1 (1988).

[66] A. Nehrkorn (CMS Collaboration), Nucl. Part. Phys. Proc. 287-288, 160 (2017).

[67] R. Akers et al. (OPAL Collaboration), Z. Phys. C 67, 555 (1995).

[68] P. Abreu et al. (DELPHI Collaboration), Z. Phys. C 73, 243 (1997).

[69] V. Khachatryan et al. (CMS Collaboration), Phys. Lett. B 763, 472 (2016).

[70] A. M. Sirunyan et al. (CMS Collaboration), J. High Energy Phys. 06 (2018) 001.

[71] P. D. Bolton, F. F. Deppisch, and P. S. B. Dev, arXiv: 1912.03058.

[72] A. Ilakovac and A. Pilaftsis, Nucl. Phys. B437, 491 (1995).

[73] W. Hollik, J. I. Illana, S. Rigolin, C. Schappacher, and D. Stockinger, Nucl. Phys. B551, 3 (1999); B557, 407(E) (1999).

[74] G. Passarino and M. J. G. Veltman, Nucl. Phys. B160, 151 (1979).

[75] T. Hahn and M. Pérez-Victoria, Comput. Phys. Commun. 118, 153 (1999).

[76] A. Denner, S. Dittmaier, and L. Hofer, Comput. Phys. Commun. 212, 220 (2017).

[77] H. H. Patel, Comput. Phys. Commun. 218, 66 (2017).

[78] A. Denner, H. Eck, O. Hahn, and J. Kublbeck, Nucl. Phys. B387, 467 (1992).

[79] E. Fernández-Martínez, J. Hernández-García, and J. LópezPavon, J. High Energy Phys. 08 (2016) 033.

[80] A. M. Coutinho, A. Crivellin, and C. A. Manzari, arXiv: 1912.08823 [Phys. Rev. Lett. (to be published)]. 
[81] R. Coy and M. Frigerio, Phys. Rev. D 99, 095040 (2019).

[82] F. del Aguila, L. Ametller, J. I. Illana, J. Santiago, P. Talavera, and R. Vega-Morales, J. High Energy Phys. 07 (2019) 154.
[83] R. Kitano, M. Koike, and Y. Okada, Phys. Rev. D 66, 096002 (2002); 76, 059902(E) (2007).

[84] T. Suzuki, D. F. Measday, and J. P. Roalsvig, Phys. Rev. C 35, 2212 (1987). 